\title{
A Severe Storm Warning System based in Radar and Satellite Data.
}

\author{
Luiz A.T. Machado, Carlos F. Angelis and Wagner F.A. Lima \\ Instituto Nacional de Pesquisas Espaciais/ Centro de Previsão de Tempo e Estudos Climáticos, INPE/CPTEC \\ Cachoeira Paulista/SP, Brasil, machado(angelis)(wagner)@cptec.inpe.br
}

\begin{abstract}
This study describes an operation tool for severe storm nowcasting using GOES satellite images and cloudground lightning data. A relationship was established between atmospheric discharges and penetrative convective clouds, combining Infrared and Water Vapor channels from the GOES-10 geostationary satellite with cloud-ground lightning data from the Brazilian Integrated Lightning Detection Network (RINDAT). The difference between water vapor and infrared brightness temperature is a tracer of penetrating clouds in the stratosphere. From this difference and the cloudground electrical discharge, it was possible to adjust exponential curves that relate this brightness temperature difference to the probability of occurrence of cloud-ground electrical discharges, with a very high coefficient of determination. These structures of penetrative clouds are followed in the time using an operational algorithm called ForTraCC (forecasting and tracking cloud cluster). This system allows extrapolating the penetrative clouds up to 120 minutes. Besides, others severity parameters were studied like the kernel of minimum cloud top temperature and the area expansion. All these information are combined in a Web based Geographic Information System called SIGMA (Geographic Information System Applied to the Environmental). This system is a useful tool to interpret and integrate the environmental informs and sends warning for emergency management groups (by e-mail or SMS).
\end{abstract}

Keywords: Nowcasting, Convective System, Precipitation

PACS: 92.60.QX

\section{Introduction.}

The Severe Storm Observation and Warning System - SOS is a tool to interpret and integrate Geographical data with environmental data and sends warning for emergency management groups (by e-mail or SMS). The SOS is composed by two components; the first one corresponds to the nowcasting tools that have input of radar, satellite, and cloud-ground lightning data. The second SOS component integrates all these information and combine in a Web based Geographic Information System called SIGMA (Geographic Information System Applied to the Environmental). This study describes the methodologies employed for the warming and nowcasting system and presents examples of the SOS Web page.

\section{Detection of Severe Weather Conditions}

The nowcasting tools are composed basically by four tolls, the ForTracc (Forecasting and Tracking Cloud Cluster), the Probability of lightning, the Hydroestimator and Radar CAPPI (Constant Altitude Plan Position Indicator) and VIL (Vertically Integrated Liquid).

\subsection{Nowcasting Satellite}

The Forecast and Tracking the evolution of Cloud Clusters technique - Fortracc is a nowcasting system based in an algorithm for tracking and forecast radiative and morphological characteristics of Mesoscale Convective Systems (MCS) through its whole life cycle using geostationary satellites thermal channel information $(10.8 \mu \mathrm{m})$. The main features of this system are the following: (1) a cloud cluster detection method based on a threshold temperature (235 $\mathrm{K}$ ); (2) a tracking technique based on MCS overlapping areas in successive images; and (3) a forecast module based on the evolution of each particular MCS in previous steps. This feature is based on the MCS possible displacement (considering the center of mass position of the cloud cluster in previous time steps) and its size evolution. Statistical information about MCS evolution was used to obtain area expansion mean rates for different MCS according to its life time duration. The ForTraCC performance can be evaluated based in the difference between forecasted and 
observed images. The evaluation shows a good agreement between the observed and forecast size and minimum temperature for shorter forecast lead times, but tends to underestimate MCS size (and overestimate the minimum temperature) for larger forecast larger than 120 minutes. An example of the Fortracc System Web page is presented in Figure 1. See Vila et all., 2008 for a detailed description.

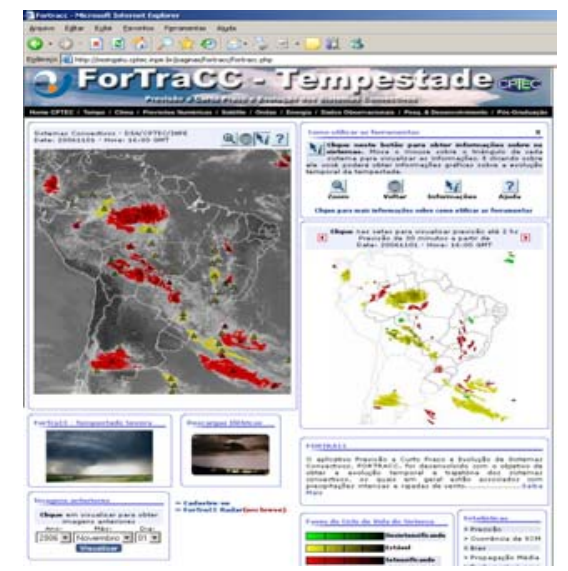

Figure 1 - Example of the ForTraCC web page - (http://moingatu.cptec.inpe.br/paginas/fortracc/fortracc.php)

\subsection{Nowcasting Radar}

The radar nowcasting is based on an adapted version of the Fortracc to use $20 \mathrm{dBZ}$ thresholds on the CAPPI 3 $\mathrm{km}$ to follow the precipitation cells and nowcasting up to 120 minutes. The same procedure is also applied to the VIL, based in the 3D data, to follow the structures of $10 \mathrm{~kg} \cdot \mathrm{m}^{-2}$, Greene and Clark (1972) shown that the fast growing of VIL is an indicator of severe storm. The $10 \mathrm{~kg} / \mathrm{m}^{2}$ threshold was chosen to follow the severe storm as suggested by Breidenbach et al. (1995). Figure 2 shows an example of the VIL structure forecast by Fortracc and the one observed.

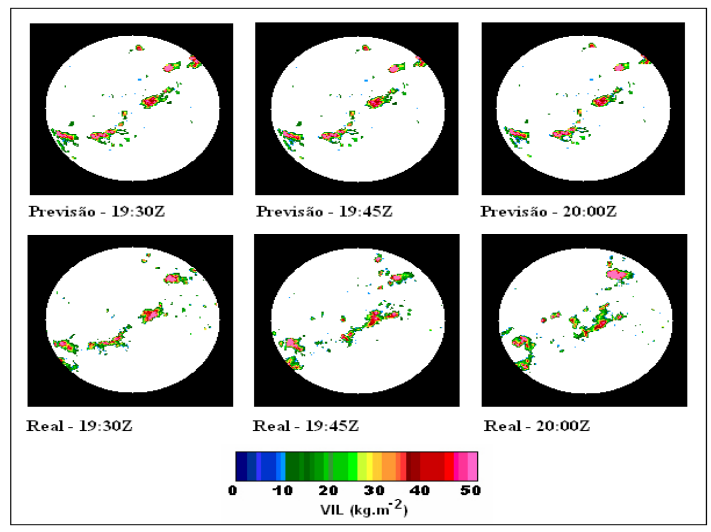

Figure 2 - The VIL structure forecast by Fortracc and observed by São Roque radar on 29 November 2006.

\subsection{Risk of lightning occurrence}

A relationship between atmospheric discharges and penetrative convective clouds was established using satellite data. It combines Infrared and Water Vapor channels from the GOES-10 geostationary satellite with cloud-ground lightning data from the Brazilian Integrated Lightning Detection Network (RINDAT). The difference between water vapor and infrared brightness temperature is a tracer penetrating clouds. Due to the water vapor channel's strong absorption, this difference is positive only during overshooting cases, when convective clouds penetrate the stratosphere. From this difference and the cloud-ground electrical discharge, measured on the ground by RINDAT, it was possible to adjust exponential curves that relate the brightness temperature difference from these two channels to the probability of occurrence of cloud-ground electrical discharges, with a very high coefficient of determination. If WV-IR brightness temperature difference is larger than $-15 \mathrm{~K}$ there is a high potential for cloud-ground lightning activity. As this difference increases the cloud-ground lightning probably increase, for example: if this difference is equal zero, the probability of having at least one cloud-ground electrical discharge is $10.9 \%, 7.0 \%$ for two, $4.4 \%$ for 
four, $2.7 \%$ for eight and $1.5 \%$ for sixteen cloud-ground lightning discharges (see Figure 3 ) Through this process, was developed a scheme that estimates the probability of occurrence of cloud-ground lightning over all the continental region of South America. An example of this product is shown in Figure 4, See Machado et al., 2008 for a detailed description

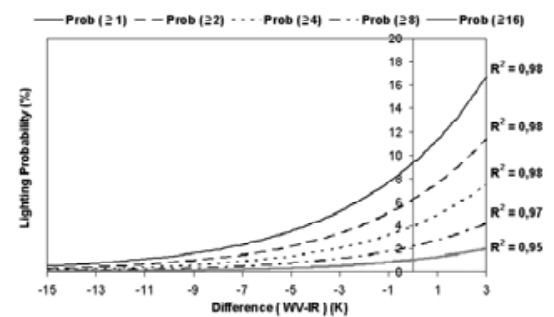

Figure 3 - Adjusted exponential curves for the probability of cloud-ground electrical discharge as a function of the WV-IR brightness temperature difference for the probability of having at least one, two, four, eight and sixteen lightning discharges in 15-minute intervals.

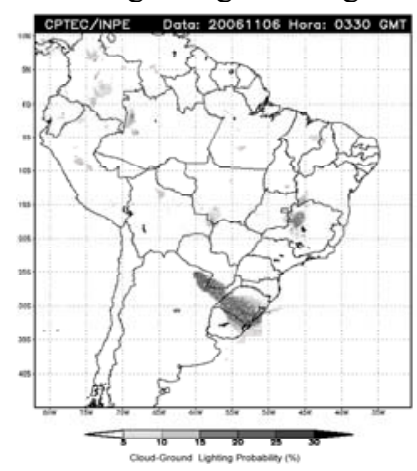

(a) ground electrical discharges per pixel 7.5 minutes before and after 03:30 GMT on the same day.

\subsection{Precipitation}

Precipitation estimation is based in the Hydroestimator using GOES 10 IR images each 15 minutes and atmosphere data from ETA model. The Hydroestimator is a technique based in Vicente et al. 1998 to compute rainfall rates. It uses a non-linear power law relationship between cloud top temperature and radar-derived rainfall rate estimates, a gradient, growth rate mask and orographic precipitation correction

Hydroestimator is not very precise to estimate rainfall in real time; however, for cumulated rainfall the results are quite good and have nearly the same precision as the 3B42 dataset. The warning system is based in the rainfall accumulation in 12,24 , and 48 hours.

\section{SOS Interface}

The product integration, visualization and analyses use Mapserver technology. MapServer's (http://mapserver.gis.umn.edu/) is an open source development environment for building spatially-enabled internet applications. MapServer excels at rendering spatial data (maps, images, and vector data) for the web. Mapserver technology is been used to create a GIS named SIGMA (GIS for Environmental Applications). For an ordinary user who searches meteorological information to plan his or her daily activities, the use of a GIS is much more than a simple "look at a single map" which usually shows static and isolated information about a specific theme. By the use of a GIS it is possible to combine a lot of information and visualize the result in form of layers which contain different data. The integration of data is one of the primary functionalities of a GIS. The SIGMA in its full conception will be a proper web based GIS developed to attend the users needs not only for basic consultation, but also for advanced operations which make use of special tools designed to obtain new information. The SOS is one of the SIGMA interfaces which appear in the web browser when the user can choose which layer and/or products they want to visualize and also see if there is an alert emitted. When a critical situation is registered, for example, if a storm is predicted to occur in one hour time, the county limit where this storm will possibly occur will be highlighted in the screen (see Figure 5). In this case, SOS can send a message to someone by email and also by mobile phone. From the SOS interface the user can see the past evolution of an extreme event and also its future 
evolution assessed by the nowcasting techniques. As the SOS is embedded in the SIGMA, all visualization can be integrated with other layers or products which are available in the SIGMA. Figure 6 illustrates a storm monitored by SOS. It combines lighting, satellite and radar data.

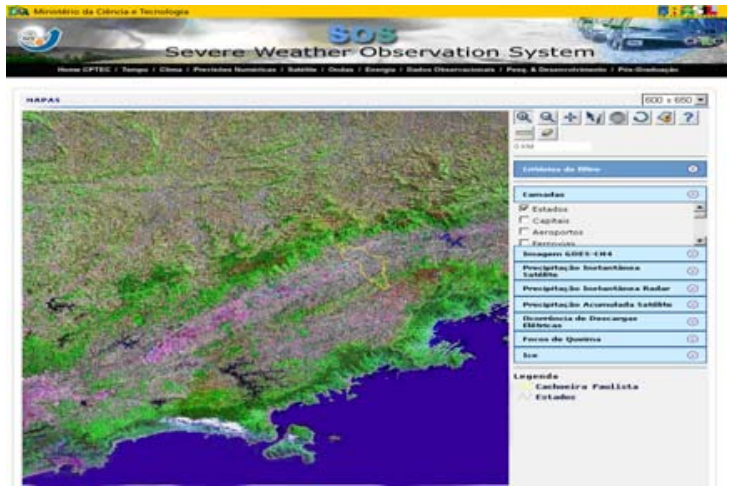

Figure 5 - SOS interface for web browser

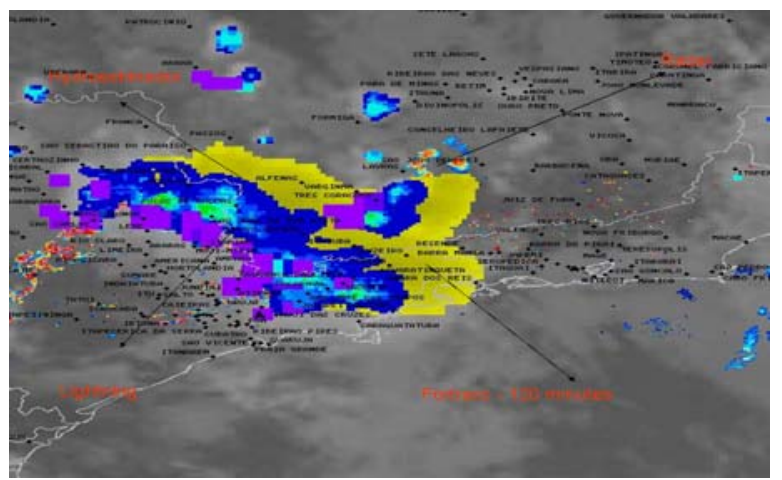

Figure 6 - Example of data integration

\section{Conclusions}

Weather conditions can have impacts on the society as a whole and are sometimes responsible for lives and economical losses. Minimizing these losses is one of the main challenges for meteorologists and climatologists because this task requires efficient automatic systems which can predict the occurrence of extreme weather events reliably. Automatic systems developed to alert about bad weather conditions must be built to help decision makers improve their capacity to let authorities know when and where an extreme event will occur. Systems like SOS and SIGMA showed above are examples of the use of GIS technology to offer users advanced tools to aid them in dealing with different types of information at the same time. The difficult development in this kind of system is the definition of the thresholds for alerts. We are starting to use the following values: area expansion larger than 200 $\mathrm{x} 10^{-6} \mathrm{~s}^{-1}$ and IR brightness temperature smaller than $210 \mathrm{~K}$, VIL larger than $10 \mathrm{~kg} \cdot \mathrm{m}^{-2}$, lightning probability larger than $15 \%$, and accumulated precipitation larger than $80 \mathrm{~mm}$. All these values should be adjusted regionally and the feedbacks of the users are of fundamental importance.

\section{REFERENCES}

Breidenbach, J. P., Kitzmiller, D. H., Mcgovern, W. E. and Saffle, R. E.: The use of volumetric radar reflectivity predictors in the development of a second-generation severe weather potential algorithm. Wea. Forecasting, 10: 269-379, 1995.

Greene, D. R., and R. A. Clark, Vertically integrated liquid water - A new analysis tool, Monthly Weather Review, 100, 548-552, 1972.

Machado, L.A.T. W. A. Lima, O. Pinto and C. A. Morales. Relationship between Cloud-Ground Lightning and Penetrative Clouds: A Multi-channel Satellite Application. Submitted to Atmospheric Research, 2008.

Vicente, G., R. A. Scofield, and W. P. Menzel, 1998: The operational GOES infrared rainfall estimation technique. Bull. Amer. Meteor. Soc., 79, 1881-1898.

Vila, Daniel, Machado, L. A. T., Laurent Henri, Velasco, Inês. Forecast and tracking the evolution of cloud cluster (ForTraCC) using satellite infrared imagery: Methodology and Validation. Weather and Forecasting. v.23, p.233 - 245, 2008. 